International Mathematical Forum, Vol. 8, 2013, no. 7, 331 - 346

\title{
On Local Controllability of Nonlinear Hyperbolic Equation
}

\section{Dieudonné Ampini, Fidèle Moukamba and Yumba Nkasa}

\author{
Département de Mathématiques \\ Faculté des Sciences \\ Université Marien NGOUABI \\ BP. 69 Brazzaville-République du Congo \\ moukambaf@yahoo.fr
}

\begin{abstract}
In this paper, we prove local controllability of a one-dimensional nonlinear wave equation, with control functions on the right hand of the eqution, using the inversion theorem.
\end{abstract}

Mathematics Subject Classification: 58J45, 35Q93, 35Lxx

Keywords: control function, parabolic equation

\section{Introduction}

In this paper, we present a result related to the following local controlability problem for the nonlinear one-dimensional wave equation. We consider the following nonlinear equation:

$$
\begin{aligned}
u_{t t}(x, t)-u_{x x}(x, t) & =F(x, t) \\
\left.u(x, t)\right|_{t=0} & =0,\left.u_{t}(x, t)\right|_{t=0}=0,0 \leq x \leq 0 \\
\left.u(x, t)\right|_{x=0} & =0,\left.u(x, t)\right|_{x=l}=0,0 \leq t \leq T \leq, 0 \leq x \leq 0 \\
\left.u(x, t)\right|_{t=T} & =\chi_{1}(x),\left.u_{t}(x, t)\right|_{t=T}=\chi_{2}(x)
\end{aligned}
$$

where $F(x, t)=\left(\frac{x}{l}-1\right) f(t)-\frac{x}{l} g(t)$.

The problem of local controlability can be formated in the following way:

in time $T>0$, we propose to drive the system from its initial state

$\left.u(x, t)\right|_{t=0}=0,\left.u_{t}(x, t)\right|_{t=0}=0$

to a given final state

$\left.u(x, t)\right|_{t=T}=\chi_{1}(x),\left.u_{t}(x, t)\right|_{t=T}=\chi_{2}(x)$

by mean of the action of control functions $f$ and $g$. 
In order words, for fixed $T>0$, find control functions $f$ and $g$ such that the solution $u$ of the equation satisfies the conditions $\left.u(x, t)\right|_{t=T}=\chi_{1}(x),\left.u_{t}(x, t)\right|_{t=T}=\chi_{2}(x)$.

To prove the existence theorem of controls, we use the properties of solutions of a linear problem and the local inversion theorem in the linear and quasilinear cases, the same results was established respectively in [1] and [2].

In nonlinear case, similar results have been established in [3].

\section{Statement of the problem and notations}

$\Gamma=] \Gamma[\subset \mathbf{R}, I=] 0, T\left[\subset \mathbf{R}, Q_{T}=\Gamma \times I\right.$.

$H^{p, m}(\Gamma)=\left\{f: \Gamma \longrightarrow \mathbf{R}: f^{(m)} \in L^{p}(\Gamma)\right\}$

$H^{m}(\Gamma)=\left\{f: \Gamma \longrightarrow \mathbf{R}: f^{(m)} \in L^{2}(\Gamma)\right\}$

$H_{0}^{1}(\Gamma)=\overline{D(\Gamma)}$ in $H^{1}(\Gamma)$.

In order to state in a rigorous way the problem, we need to remember some results established in [1] and [2].

Let $u=u(x, t)$. Consider the problem

$$
\begin{array}{rcc}
u_{t t}-u_{x x}= & F(x, t) \\
\left.u\right|_{t=0}= & 0,\left.u_{t}\right|_{t=0}=0,0 \leq x \leq l \\
\left.u\right|_{x=0}= & 0,\left.u\right|_{x=l}=0, \quad 0 \leq l \leq T \\
\left.u(x, t)\right|_{t=T}= & \chi_{1}(x),\left.u_{t}(x, t)\right|_{t=T}=\chi_{2}(x)
\end{array}
$$

where $F(x, t)=\left(\frac{x}{l}-1\right) f(t)-\frac{x}{l} g(t)$.

$\chi_{1} \in H^{5}(\Gamma) \cap H_{0}^{1}(\Gamma), \chi_{2} \in H^{4}(\Gamma) \cap H_{0}^{1}(\Gamma), f \in H^{3}(I), g \in H^{3}(I)$.

Definition 1 ([1], p.693) . We say that a function $(x, t) \longmapsto u(x, t)$ belongs to class $W_{2}^{2}\left(Q_{T}\right)$, if $u$ and its first partial derivates are continuous in $\bar{Q}_{T}$, and if this function admits all second generalised partial dervatives belonging to $L_{2}(\Gamma)$ for all $t \in I$, and to $L^{2}(I)$ for all $x \in \bar{\Gamma}$.

Definition $2\left([\mathbf{1}]\right.$, p.694) . $(u, f, g) \in W_{2}^{2}\left(Q_{T}\right) \times H^{3}(I) \times H^{3}(I)$ is said to be a solution of the mixed problem (1) - (4), if:

(i) $(u, f, g)$ satisfies the equation (1) a.e. in $Q_{T}$;

(ii) $u$ satisfies the initial conditions (3) a.e. in $\bar{\Gamma}$;

(iii) $u$ satisfies the boundary conditions (3) a. e. in $\bar{I}$;

(iv) $u$ satisfies the conditions (4) a.e. in $\bar{\Gamma}$.

Theorem 1 ([2], p. 402) . There exists a unique solution for which u is a solution of the problem (1) - (4).

Remark 1 Let $H=\left\{u \in W_{2}^{2}\left(Q_{T}\right) / \exists F(x, t) \in H^{3}\left(Q_{T}\right):(u, f, g)\right.$ is a solution of the mixed problem $(1)-(4)\}$, 
with the norm $\|u\|_{H}=\|L u\|_{H^{3}\left(Q_{T}\right)}$,

where $L=\frac{\partial^{2}}{\partial t^{2}}-\frac{\partial^{2}}{\partial x^{2}}$.

Then $L: H \longrightarrow H^{3}\left(Q_{T}\right)$ is an isomorphism. To prove this fact, see ([4] P. 306). Note that

$H \subset\left\{u \in W_{2}^{2}\left(Q_{T}\right) /\left.u(x, t)\right|_{t=0}=0,\left.u_{t}(x, t)\right|_{t=0}=0,\left.u(x, t)\right|_{x=0}=0,\left.u(x, t)\right|_{x=0}=0\right\}$. This implies that $\left(H,\|.\|_{H}\right)$ is a complete space. We also get the continuous embedding $H \hookrightarrow H^{4}\left(Q_{T}\right)$. This means that $H \subset H^{4}\left(Q_{T}\right)$.

\section{Formulation of the main result}

Let $u \longmapsto \nu(u) \in C^{4}(\mathbf{R})$; with a function of two real variables, $x \in \Gamma$ and $t \in I$, such that:

$$
\nu(0)=0, \nu^{\prime}(0)=0
$$

Consider the problem

$$
\begin{aligned}
u_{t t}-u_{x x}+\nu(u(x, t) & =F(x, t) \\
(6) & \\
\left.u\right|_{t=0}=0,\left.u_{t}\right|_{t=0} & =0,0 \leq x \leq l \\
(7) & \\
\left.u\right|_{x=0}=0,\left.u\right|_{x=l} & =0,0 \leq l \leq T \\
(8) & \\
\left.u(x, t)\right|_{t=T} & =\chi_{1}(x),\left.u_{t}(x, t)\right|_{t=T}=\chi_{2}(x)
\end{aligned}
$$

where $F(x, t)=\left(\frac{x}{l}-1\right) f(t)-\frac{x}{l} g(t)$.

The main result of this paper is the following.

Theorem 2 If conditions (5) are satified, then there exits a unique solution $(u, f, g)$ on a neighborhood of zero in $H^{4}\left(Q_{T}\right) \times H^{3}(I) \times H^{4}(I)$ for small enough $\left(\chi_{1}, \chi_{2}\right) \in$ $H^{5}(\Gamma) \cap H_{0}^{1}(\Gamma) \times H^{4}(\Gamma) \cap H_{0}^{1}(\Gamma)$.

Remark 2 In order to prove theorem 2, we have to verify the fact that the conditions of the inversion theorem are satisfied; which means that we must establish the differentiability of the involved mappings and specify the functional spaces in which we are going to study problem (6) - (9).

\section{Differentiability of Nemwisk operator}

Proposition 3 The operator $N: H^{4}\left(Q_{T}\right) \longrightarrow H^{3}\left(Q_{T}\right), u \longmapsto N(u)$, where $[N(u)](x, t)=$ $\nu(u(x, t))$ is differentiable in $H^{4}\left(Q_{T}\right)$ and

$$
\left[N^{\prime}(u) \hat{u}\right](x, t)=\nu^{\prime}(u(x, t)) \hat{u}(x, t) .
$$


Proof. First of all, let us prove that the operator $N$ mapps $H^{4}\left(Q_{T}\right)$ into $H^{3}\left(Q_{T}\right)$.Suppose that $u \in H^{4}\left(Q_{T}\right)$. Then according to the embedding theorem, we have

$$
u \in C\left(\bar{Q}_{T}\right), \quad u_{t} \in H^{3}\left(Q_{T}\right) \subset C\left(\bar{Q}_{T}\right), \quad u_{t t} \in H^{2}\left(Q_{T}\right) \subset C\left(\bar{Q}_{T}\right)
$$

and

$$
u_{x} \in H^{3}\left(Q_{T}\right) \subset C\left(\bar{Q}_{T}\right), \quad u_{x x} \in H^{2}\left(Q_{T}\right) \subset C\left(\bar{Q}_{T}\right) .
$$

In this case $\int_{Q_{T}}|\nu(u(x, t))|^{2} d x d t<\infty$ since $\nu(u) \in C\left(Q_{T}\right)$, which means that $\nu(u(x, t)) \in L_{2}\left(Q_{T}\right)$. We have

$$
\begin{aligned}
\int_{Q_{T}}\left|\frac{\partial \nu}{\partial t}(u(x, t))\right|^{2} d x d t & =\int_{Q_{T}}\left|\nu^{\prime}(u(x, t)) u_{t}\right|^{2} d x d t \\
& \leq \sup _{(x, t) \in Q_{T}}\left|\nu^{\prime}(u)\right|^{2} \int_{Q_{T}}\left|u_{t}(x, t)\right|^{2} d x d t \\
& =\sup _{(x, t) \in Q_{T}}\left|\nu^{\prime}(u)\right|^{2}\left\|u_{t}\right\|_{L_{2}\left(Q_{T}\right)}^{2}<\infty,
\end{aligned}
$$

i.e. $\frac{\partial \nu}{\partial t}(u(x, t)) \in L_{2}\left(Q_{T}\right)$.In the same way, we can estimate $\int_{Q_{T}}\left|\frac{\partial \nu}{\partial x}(u(x, t))\right|^{2} d x d t$. We then have:

$$
\begin{aligned}
& \int_{Q_{T}}\left|\frac{\partial^{2} \nu}{\partial t^{2}}(u(x, t))\right|^{2} d x d t \\
= & \int_{Q_{T}} \mid \frac{\partial}{\partial t}\left(\left.\frac{\partial \nu}{\partial t}(u(x, t))\right|^{2} d x d t\right. \\
= & \int_{Q_{T}}\left|\frac{\partial \nu^{\prime}}{\partial t}(u(x, t)) u_{t}(x, t)\right|^{2} d x d t \\
= & \int_{Q_{T}}\left|\nu^{\prime \prime}(u(x, t)) u_{t}^{2}(x, t)+\nu^{\prime}(u(x, t)) u_{t t}(x, t)\right|^{2} d x d t \\
\leq & 2 \int_{Q_{T}}\left|\nu^{\prime \prime}(u(x, t)) u_{t}^{2}(x, t)\right|^{2} d x d t+2 \int_{Q_{T}}\left|\nu^{\prime}(u(x, t)) u_{t t}(x, t)\right|^{2} d x d t \\
\leq & 2 \sup _{(x, t) \in Q_{T}}\left|\nu^{\prime \prime}(u)\right|^{2} \int_{Q_{T}}\left|u_{t}(x, t)\right|^{2} d x d t+2 \sup _{(x, t) \in Q_{T}}\left|\nu^{\prime}(u)\right|^{2} \int_{Q_{T}}\left|u_{t t}(x, t)\right|^{2} d x d t \\
= & 2 \sup _{(x, t) \in Q_{T}}\left|\nu^{\prime \prime}(u)\right|^{2}\left\|u_{t}\right\|_{L_{4}\left(Q_{T}\right)}^{4}+2 \sup _{(x, t) \in Q_{T}}\left|\nu^{\prime}(u)\right|^{2}\left\|u_{t t}\right\|_{L_{2}\left(Q_{T}\right)}^{2}<\infty
\end{aligned}
$$


i.e. $\frac{\partial^{2} \nu}{\partial t^{2}}(u(x, t)) \in L_{2}\left(Q_{T}\right)$. Similarly, we can estimate $\int_{Q_{T}}\left|\frac{\partial^{2} \nu}{\partial x^{2}}(u(x, t))\right|^{2} d x d t$. Next,

$$
\begin{aligned}
& \int_{Q_{T}}\left|\frac{\partial^{2} \nu}{\partial x \partial t}(u(x, t))\right|^{2} d x d t \\
= & \int_{Q_{T}} \mid \frac{\partial}{\partial x}\left(\left.\frac{\partial \nu}{\partial t}(u(x, t))\right|^{2} d x d t\right. \\
= & \int_{Q_{T}}\left|\frac{\partial}{\partial x}\left(\nu^{\prime}(u(x, t)) u_{t}(x, t)\right)\right|^{2} d x d t \\
= & \int_{Q_{T}}\left|\nu^{\prime \prime}(u(x, t)) u_{x}(x, t) u_{t}(x, t)+\nu^{\prime}(u(x, t)) u_{t x}(x, t)\right|^{2} d x d t \\
\leq & 2 \int_{Q_{T}} \mid \nu^{\prime \prime}(u(x, t)) u_{x}\left(x,\left.t u_{t}(x, t)\right|^{2} d x d t+2 \int_{Q_{T}}\left|\nu^{\prime}(u(x, t)) u_{t x}(x, t)\right|^{2} d x d t\right. \\
\leq & 2 \sup _{(x, t) \in Q_{T}}\left|\nu^{\prime \prime}(u)\right|^{2}\left(\int_{Q_{T}}\left|u_{x}(x, t)\right|^{4} d x d t\right)^{\frac{1}{2}}\left(\int_{Q_{T}}\left|u_{t}(x, t)\right|^{4} d x d t\right)^{\frac{1}{2}} \\
& +2 \sup _{(x, t) \in Q_{T}}\left|\nu^{\prime}(u)\right|^{2} \int_{Q_{T}}\left|u_{t x}(x, t)\right|^{2} d x d t .
\end{aligned}
$$

Thus

$$
\begin{aligned}
& \int_{Q_{T}}\left|\frac{\partial^{2} \nu}{\partial x \partial t}(u(x, t))\right|^{2} d x d t \leq 2 \sup _{(x, t) \in Q_{T}}\left|\nu^{\prime \prime}(u)\right|^{2}\left\|u_{x}\right\|_{L_{4}\left(Q_{T}\right)}^{2}\left\|u_{t}\right\|_{L_{4}\left(Q_{T}\right)}^{2} \\
& +2 \sup _{(x, t) \in Q_{T}}\left|\nu^{\prime}(u)\right|^{2}\left\|u_{t t}\right\|_{L_{2}\left(Q_{T}\right)}^{2}<+\infty
\end{aligned}
$$

i.e

$$
\frac{\partial^{2} \nu}{\partial x \partial t}(u(x, t)) \in L_{2}\left(Q_{T}\right)
$$

Similarly, we can estimate $\int_{Q_{T}}\left|\frac{\partial^{2}}{\partial t \partial x} \nu(u(x, t))\right|^{2} d x d t$.

Finaly, let us estimate $\int_{Q_{T}}\left|\frac{\partial^{3}}{\partial t^{3}} \nu(u(x, t))\right|^{2} d x d t$ 


$$
\begin{aligned}
& \int_{Q_{T}}\left|\frac{\partial^{3}}{\partial t^{3}} \nu(u(x, t))\right|^{2} d x d t \\
= & \int_{Q_{T}} \mid \frac{\partial}{\partial t}\left(\left.\frac{\partial^{2} \nu}{\partial t^{2}}(u(x, t))\right|^{2} d x d t\right. \\
= & \int_{Q_{T}} \mid \frac{\partial}{\partial t}\left[\nu^{\prime \prime}(u(x, t)) u_{t}^{2}(x, t)+\left.\nu^{\prime}(u(x, t)) u_{t t}(x, t]\right|^{2} d x d t\right. \\
= & \int_{Q_{T}}\left|\nu^{\prime \prime \prime}(u) u_{t}^{3}+3 \nu^{\prime \prime}(u) u_{t} u_{t t}+\nu^{\prime}(u) u_{t t t}\right|^{2} d x d t \\
\leq & 3 \sup _{Q_{T}}\left|\nu^{\prime \prime \prime}(u)\right|^{2} \int_{Q_{T}} u_{t}^{6} d x d t+3 \sup _{Q_{T}}\left|\nu^{\prime \prime}(u)\right|^{2} \int_{Q_{T}} u_{t}^{2} u_{t t}^{2} d x d t \\
& \left.+\sup _{Q_{T}}\left|\nu^{\prime}(u)\right|^{2} \int_{Q_{T}} u_{t t t}^{2} d x d t\right) .
\end{aligned}
$$

This implies

$$
\begin{aligned}
\int_{Q_{T}}\left|\frac{\partial^{3} \nu}{\partial t^{3}}(u(x, t))\right|^{2} d x d t \leq & 3 \sup _{(x, t) \in Q_{T}}\left|\nu^{\prime \prime \prime}(u)\right|^{2}\left\|u_{t}\right\|_{L_{6}\left(Q_{T}\right)}^{6} \\
& +3 \sup _{(x, t) \in Q_{T}}\left|\nu^{\prime \prime}(u)\right|^{2}\left\|u_{t}\right\|_{L_{4}\left(Q_{T}\right)}^{2}\left\|u_{t t}\right\|_{L_{4}\left(Q_{T}\right)}^{2} \\
& +\sup _{(x, t) \in Q_{T}}\left|\nu^{\prime}(u)\right|^{2}\left\|u_{t t t}\right\|_{L_{2}\left(Q_{T}\right)}^{2}\left\|u_{t}\right\|_{L_{4}\left(Q_{T}\right)}^{2}<+\infty
\end{aligned}
$$

i.e.

$$
\frac{\partial^{3}}{\partial t^{3}} \nu(u(x, t)) \in L_{2}\left(Q_{T}\right)
$$

Similarly, we can estimate $\int_{Q_{T}}\left|\frac{\partial^{3} \nu}{\partial x^{3}}(u(x, t))\right|^{2} d x d t$.

We can, in the same way, estimate the following expressions:

$$
\begin{aligned}
& \int_{Q_{T}} \mid \frac{\partial}{\partial x}\left(\left.\frac{\partial^{2} \nu}{\partial t^{2}}(u(x, t))\right|^{2} d x d t \quad, \quad \int_{Q_{T}} \mid \frac{\partial}{\partial t}\left(\left.\frac{\partial^{2} \nu}{\partial x^{2}}(u(x, t))\right|^{2} d x d t\right.\right. \\
& \int_{Q_{T}} \mid \frac{\partial^{2}}{\partial x^{2}}\left(\left.\frac{\partial \nu}{\partial t}(u(x, t))\right|^{2} d x d t, \quad \int_{Q_{T}} \mid \frac{\partial^{2}}{\partial t^{2}}\left(\left.\frac{\partial \nu}{\partial x}(u(x, t))\right|^{2} d x d t,\right.\right. \\
& \int_{Q_{T}} \mid \frac{\partial}{\partial x}\left(\left.\frac{\partial}{\partial t}\left(\frac{\partial \nu}{\partial x}(u(x, t))\right)\right|^{2} d x d t \quad, \quad \int_{Q_{T}} \mid \frac{\partial}{\partial t}\left(\left.\frac{\partial}{\partial x}\left(\frac{\partial \nu}{\partial t}(u(x, t))\right)\right|^{2} d x d t .\right.\right.
\end{aligned}
$$

All these results together with (11) and (12) imply that $N(u) \in H^{3}\left(Q_{T}\right)$.

Now suppose that $u, u_{k}, \hat{u}_{k} \in H^{4}\left(Q_{T}\right), \hat{u}_{k} \neq 0, u_{k} \rightarrow u$ and $\hat{u}_{k} \rightarrow 0$ in $H^{4}\left(Q_{T}\right)$, when $k \rightarrow \infty$. 
Let

$$
\left[R\left(u_{k}, \hat{u}_{k}\right)\right](x, t)=\nu\left(u_{k}(x, t)+\hat{u}_{k}(x, t)\right)-\nu\left(u_{k}(x, t)\right)-\nu^{\prime}(u(x, t)) \hat{u}_{k}(x, t)
$$

and let us prove that

$$
\lim _{k \rightarrow \infty} \frac{R\left(u_{k}, \hat{u}_{k}\right)}{\left\|\hat{u}_{k}\right\|_{H^{4}\left(Q_{T}\right)}} \rightarrow 0
$$

in $H^{3}\left(Q_{T}\right)$.

Considering subsequences, we can assume that $u_{k} \rightarrow u, \hat{u}_{k} \rightarrow 0$,

$u_{k t} \rightarrow u_{t}, u_{k t t} \rightarrow u_{t t}, \hat{u}_{k t} \rightarrow 0$ and $\hat{u}_{k t t} \rightarrow 0$ almost everywhere on $Q_{T}$. Since $u \mapsto \nu^{\prime}(u)$ is continuous, $u \mapsto \nu^{\prime}(u)$ is uniformly continuous on every segment. Moreover, since $u_{k} \rightarrow u$ in $H^{4}\left(Q_{T}\right)$, from (11), (12) we get $u_{k} \rightarrow u, \hat{u}_{k} \rightarrow 0$ in $C\left(\bar{Q}_{T}\right)$. This implies that there exist constants $C_{16}$ and $C_{17}$ such that $\left\|u_{k}\right\|_{C\left(\bar{Q}_{T}\right)} \leq C_{16}$ and $\left\|\hat{u}_{k}\right\|_{C\left(\bar{Q}_{T}\right)} \leq C_{17}$.

Thus,

$$
\begin{aligned}
& \int_{Q_{T}}\left|R\left(u_{k}, \hat{u}_{k}\right)\right|^{2} d x d t \\
= & \int_{Q_{T}}\left|\nu\left(u_{k}+\hat{u}_{k}\right)-\nu\left(u_{k}\right)-\nu^{\prime}(u) \hat{u}_{k}\right|^{2} d x d t \\
= & \int_{Q_{T}}\left(\int_{0}^{1}\left[\nu^{\prime}\left(u_{k}+\theta \hat{u}_{k}\right)-\nu^{\prime}(u)\right] \hat{u}_{k} \cdot 1 . d \theta\right)^{2} d x d t \\
\leq & \int_{Q_{T}} \int_{0}^{1}\left[\nu^{\prime}\left(u_{k}+\theta \hat{u}_{k}\right)-\nu^{\prime}(u)\right]^{2} \hat{u}_{k}^{2} d \theta \cdot \int_{0}^{1} 1^{2} d \theta d x d t \\
= & \int_{0}^{1} \int_{Q_{T}}\left[\nu^{\prime}\left(u_{k}+\theta \hat{u}_{k}\right)-\nu^{\prime}(u)\right]^{2} \hat{u}_{k}^{2} d x d t d \theta \\
\leq & \sup _{(x, t) \in Q_{T}}\left|\hat{u}_{k}\right|^{2} \int_{0}^{1}\left(\int_{Q_{T}}\left|\nu^{\prime}\left(u_{k}+\theta \hat{u}_{k}\right)-\nu^{\prime}(u)\right|^{2} d x d t\right) d \theta \\
= & \left\|\hat{u}_{k}\right\|_{C}^{2}\left(\overline{Q_{T}}\right) \int_{0}^{1}\left(\int_{Q_{T}}\left|\nu^{\prime}\left(u_{k}+\theta \hat{u}_{k}\right)-\nu^{\prime}(u)\right|^{2} d x d t\right) d \theta \\
\leq & \left\|\hat{u}_{k}\right\|_{H^{4}\left(Q_{T}\right)}^{2} C_{18}^{2} \int_{0}^{1}\left(\int_{Q_{T}}\left|\nu^{\prime}\left(u u_{k}+\theta \hat{u}_{k}\right)-\nu^{\prime}(u)\right|^{2} d x d t\right) d \theta .
\end{aligned}
$$

We can see that

$$
\left|\nu^{\prime}\left(u_{k}+\theta \hat{u}_{k}\right)-\nu^{\prime}(u)\right|^{2} \rightarrow 0
$$


almost everywhere on $Q_{T}$ and that

$$
\left|\nu^{\prime}\left(u_{k}+\theta \hat{u}_{k}\right)-\nu^{\prime}(u)\right|^{2} \leq C_{18}
$$

uniformly with respect to $\theta \in[0,1]$. Using twice Lebesgue theorem, we obtain:

$$
\lim _{k \rightarrow \infty} \frac{\left\|R\left(u_{k}, \hat{u}_{k}\right)\right\|_{L_{2}\left(Q_{T}\right)}}{\left\|\hat{u}_{k}\right\|_{H^{4}\left(Q_{T}\right)}} \rightarrow 0
$$

Next, using the inequality $(a+b+c)^{2} \leq 3\left(a^{2}+b^{2}+c^{2}\right)$, we have

$$
\begin{aligned}
& \int_{Q_{T}}\left|\frac{\partial}{\partial t}\left[R\left(u_{k}, \hat{u}_{k}\right)\right](x, t)\right|^{2} d x d t \\
& =\int_{Q_{T}}\left[\frac{\partial}{\partial t}\left(\nu\left(u_{k}+\hat{u}_{k}\right)-\nu\left(u_{k}\right)-\nu^{\prime}(u) \hat{u}_{k}\right)\right]^{2} d x d t \\
& =\int_{Q_{T}}\left(\frac{\partial}{\partial t} \int_{0}^{1}\left[\nu^{\prime}\left(u_{k}+\theta \hat{u}_{k}\right)-\nu^{\prime}(u)\right] \hat{u}_{k} d \theta\right)^{2} d x d t \\
& =\int_{Q_{T}}\left(\int _ { 0 } ^ { 1 } \left\{\left[\nu^{\prime \prime}\left(u_{k}+\theta \hat{u}_{k}\right)\left(u_{k t}+\theta \hat{u}_{k t}\right)-\nu^{\prime \prime}(u) u_{t}\right] \hat{u}_{k}\right.\right. \\
& \left.\left.+\left[\nu^{\prime}\left(u_{k}+\theta \hat{u}_{k}\right)-\nu^{\prime}(u)\right] \hat{u}_{k t}\right\} d \theta\right)^{2} d x d t \\
& =\int_{Q_{T}}\left(\int _ { 0 } ^ { 1 } \left\{\left[\nu^{\prime \prime}\left(u_{k}+\theta \hat{u}_{k}\right)-\nu^{\prime \prime}(u)\right]\left(u_{k t}+\theta \hat{u}_{k t}\right) \hat{u}_{k}\right.\right. \\
& +\nu^{\prime \prime}(u)\left(u_{k t}+\theta \hat{u}_{k t}-u_{t}\right) \hat{u}_{k} \\
& \left.\left.+\left[\nu^{\prime}\left(u_{k}+\theta \hat{u}_{k}\right)-\nu^{\prime}(u)\right] \hat{u}_{k t}\right\} d \theta\right)^{2} d x d t \\
& \leq \int_{Q_{T}} \int_{0}^{1}\{\ldots\}^{2} d \theta d x d t=\int_{0}^{1} \int_{Q_{T}}\{\ldots\}^{2} d x d t d \theta \\
& \leq 3\left[\int_{0}^{1} \int_{Q_{T}}\left|\nu^{\prime \prime}\left(u_{k}+\theta \hat{u}_{k}\right)-\nu^{\prime \prime}(u)\right|^{2}\left|u_{k t}+\theta \hat{u}_{k t}\right|^{2} \hat{u}_{k}^{2} d x d t d \theta\right. \\
& +\int_{0}^{1} \int_{Q_{T}}\left|\nu^{\prime \prime}(u)\right|^{2}\left|\left(u_{k t}+\theta \hat{u}_{k t}-u_{t}\right)\right|^{2} \hat{u}_{k}^{2} d x d t d \theta \\
& \left.+\int_{0}^{1} \int_{Q_{T}}\left|\nu^{\prime}\left(u_{k}+\theta \hat{u}_{k}\right)-\nu^{\prime}(u)\right|^{2} \hat{u}_{k t}^{2} d x d t d \theta\right]
\end{aligned}
$$




$$
\begin{aligned}
& \leq 3\left[\left\|\hat{u}_{k}\right\|_{C\left(Q_{T}\right)}^{2} \int_{0}^{1} \int_{Q_{T}}\left|\nu^{\prime \prime}\left(u_{k}+\theta \hat{u}_{k}\right)-\nu^{\prime \prime}(u)\right|^{2}\left|u_{k t}+\theta \hat{u}_{k t}\right|^{2} d x d t d \theta\right. \\
& +\left\|\hat{u}_{k}\right\|_{C\left(Q_{T}\right)}^{2} \int_{0}^{1} \int_{Q_{T}}\left|\nu^{\prime \prime}(u)\right|^{2}\left|\left(u_{k t}+\theta \hat{u}_{k t}-u_{t}\right)\right|^{2} d x d t d \theta \\
& \left.+\int_{0}^{1} \int_{Q_{T}}\left|\nu^{\prime}\left(u_{k}+\theta \hat{u}_{k}\right)-\nu^{\prime}(u)\right|^{2} \hat{u}_{k t}^{2} d x d t d \theta\right] \\
& \leq 3\left[\left\|\hat{u}_{k}\right\|_{C\left(Q_{T}\right)}^{2} \int_{0}^{1}\left(\int_{Q_{T}}\left|\nu^{\prime \prime}\left(u_{k}+\theta \hat{u}_{k}\right)-\nu^{\prime \prime}(u)\right|^{4} d x d t\right)^{\frac{1}{2}}\right. \\
& +\left(\int_{Q_{T}}\left|u_{k t}+\theta \hat{u}_{k t}\right|^{4} d x d t\right)^{\frac{1}{2}} d \theta+\left\|\hat{u}_{k}\right\|_{C\left(Q_{T}\right)}^{2} \\
& +\int_{0}^{1}\left(\int_{Q_{T}}\left|\nu^{\prime \prime}(u)\right|^{4} d x d t\right)^{\frac{1}{2}}\left(\int_{Q_{T}}\left|\left(u_{k t}+\theta \hat{u}_{k t}-u_{t}\right)\right|^{4} d x d t\right)^{\frac{1}{2}} d \theta \\
& \left.+\int_{0}^{1}\left(\int_{Q_{T}}\left|\nu^{\prime}\left(u_{k}+\theta \hat{u}_{k}\right)-\nu^{\prime}(u)\right|^{4} d x d t\right)^{\frac{1}{2}}\left(\int_{Q_{T}} \hat{u}_{k t}^{4} d x d t\right)^{\frac{1}{2}} d \theta\right] \\
& \leq C_{19}\left[\int_{0}^{1}\left(\int_{Q_{T}}\left|\nu^{\prime \prime}\left(u_{k}+\theta \hat{u}_{k}\right)-\nu^{\prime \prime}(u)\right|^{4} d x d t\right)^{\frac{1}{2}} d \theta\left\|\hat{u}_{k}\right\|_{C\left(Q_{T}\right)}^{2}\right. \\
& +\left\|\hat{u}_{k}\right\|_{C\left(Q_{T}\right)}^{2} \int_{0}^{1}\left(\int_{Q_{T}}\left|\left(u_{k t}+\theta \hat{u}_{k t}-u_{t}\right)\right|^{4} d x d t\right)^{\frac{1}{2}} d \theta \\
& \left.+\int_{0}^{1}\left(\int_{Q_{T}}\left|\nu^{\prime}\left(u_{k}+\theta \hat{u}_{k}\right)-\nu^{\prime}(u)\right|^{4} d x d t\right)^{\frac{1}{2}}\left(\int_{Q_{T}} \hat{u}_{k t}^{4} d x d t\right)^{\frac{1}{2}} d \theta\right] \\
& =C_{19}\left[\int_{0}^{1}\left(\int_{Q_{T}}\left|\nu^{\prime \prime}\left(u_{k}+\theta \hat{u}_{k}\right)-\nu^{\prime \prime}(u)\right|^{4} d x d t\right)^{\frac{1}{2}} d \theta\left\|\hat{u}_{k}\right\|_{C\left(Q_{T}\right)}^{2}\right. \\
& +\left\|\hat{u}_{k}\right\|_{C\left(Q_{T}\right)}^{2} \int_{0}^{1}\left(\int_{Q_{T}}\left|\left(u_{k t}+\theta \hat{u}_{k t}-u_{t}\right)\right|^{4} d x d t\right)^{\frac{1}{2}} d \theta \\
& \left.+\int_{0}^{1}\left(\int_{Q_{T}}\left|\nu^{\prime}\left(u_{k}+\theta \hat{u}_{k}\right)-\nu^{\prime}(u)\right|^{4} d x d t\right)^{\frac{1}{2}} d \theta\left\|\hat{u}_{k t}\right\|_{L_{4}\left(Q_{T}\right)}^{2}\right]
\end{aligned}
$$

Let us note that, in view of (11) and (12) in all of three obtained expressions, the last term can be estimated by $\left\|\hat{u}_{k}\right\|_{H^{4}\left(Q_{T}\right)}^{2}$. In view of Lebesgue theorem, the other terms are integrals converging to zero when $\mathrm{k} \rightarrow \infty$. 
Indeed, $\left|\nu^{\prime \prime \prime}\left(u_{k}+\theta \hat{u}_{k}\right)-\nu^{\prime \prime \prime}(u)\right|^{4} \rightarrow 0$ almost everywhere with respect to $(x, t) \in$ $Q_{T}$ and

$$
\left|\nu^{\prime \prime}\left(u_{k}+\theta \hat{u}_{k}\right)-\nu^{\prime \prime}(u)\right|^{4} \leq C_{20}
$$

unifomly with respect to $\theta \in[0,1]$. In view of (11) and (15),

$$
\left|\left(u_{k t}+\theta \hat{u}_{k t}\right)^{2}-u_{t}^{2}\right|^{4} \rightarrow 0
$$

a.e. with respect to $(x, t) \in Q_{T}$ and

$$
\left|\left(u_{k t}+\theta \hat{u}_{k t}\right)^{2}-u_{t}^{2}\right|^{4} \in L_{1}\left(Q_{T}\right)
$$

unifomly with respect to $\theta \in[0,1]$. In view of (11) and (15),

$$
\left|\nu^{\prime \prime}\left(u_{k}+\theta \hat{u}_{k}\right)-\nu^{\prime \prime}(u)\right|^{4} \rightarrow 0
$$

a.e. with respect to $(x, t) \in Q_{T}$ and

$$
\left|\nu^{\prime \prime}\left(u_{k}+\theta \hat{u}_{k}\right)-\nu^{\prime \prime}(u)\right|^{4} \leq C_{21}
$$

unifomly with respect to $\theta \in[0,1]$.

Similarly $\int_{Q_{T}}\left|\frac{\partial}{\partial x}\left[R\left(u_{k}, \hat{u}_{k}\right)\right](x, t)\right|^{2} d x d t$ can be estimated.

We next have:

$$
\begin{aligned}
& \int_{Q_{T}}\left|\frac{\partial^{2}}{\partial t^{2}}\left[R\left(u_{k}, \hat{u}_{k}\right)\right](x, t)\right|^{2} d x d t \\
= & \int_{Q_{T}}\left\{\frac{\partial}{\partial t}\left(\frac{\partial}{\partial t}\left(R\left(u_{k}, \hat{u}_{k}\right)\right)\right)(x, t)\right\}^{2} d x d t \\
= & \int_{Q_{T}}\left(\frac { \partial } { \partial t } \int _ { 0 } ^ { 1 } \left\{\left[\left(\nu^{\prime \prime}\left(u_{k}+\theta \hat{u}_{k}\right)\left(u_{k t}+\theta \hat{u}_{k t}\right)-\nu^{\prime \prime}(u) u_{t}\right] \hat{u}_{k}\right.\right.\right. \\
& \left.\left.+\left[\nu^{\prime}\left(u_{k}+\theta \hat{u}_{k}\right)-\nu^{\prime}(u)\right] \hat{u}_{k t}\right\} d \theta\right)^{2} d x d t \\
= & \int_{Q_{T}}\left(\int _ { 0 } ^ { 1 } \left\{\left[\nu^{\prime \prime \prime}\left(u_{k}+\theta \hat{u}_{k}\right)\left(u_{k t}+\theta \hat{u}_{k t}\right)^{2}\right.\right.\right. \\
& +\left(\nu^{\prime \prime}\left(u_{k}+\theta \hat{u}_{k}\right)\left(u_{k t t}+\theta \hat{u}_{k t t}\right)-\nu^{\prime \prime \prime}(u) u_{t}^{2}-\nu^{\prime \prime}(u) u_{t t}\right] \hat{u}_{k} \\
& +\left[\left(\nu^{\prime \prime}\left(u_{k}+\theta \hat{u}_{k}\right)\left(u_{k t}+\theta \hat{u}_{k t}\right)-\nu^{\prime \prime}(u) u_{t}\right] \hat{u}_{k t}\right. \\
& +\left[\left(\nu^{\prime \prime}\left(u_{k}+\theta \hat{u}_{k}\right)\left(u_{k t}+\theta \hat{u}_{k t}\right)-\nu^{\prime \prime}(u) u_{t}\right] \hat{u}_{k t}\right. \\
& \left.\left.+\left[\nu^{\prime}\left(u_{k}+\theta \hat{u}_{k}\right)-\nu^{\prime}(u)\right] \hat{u}_{k t t}\right\} d \theta\right)^{2} d x d t \\
= & \int_{Q_{T}}\left(\int _ { 0 } ^ { 1 } \left\{\left[\nu^{\prime \prime \prime}\left(u_{k}+\theta \hat{u}_{k}\right)\left(u_{k t}+\theta \hat{u}_{k t}\right)^{2}\right.\right.\right. \\
& +\left(\nu^{\prime \prime}\left(u_{k}+\theta \hat{u}_{k}\right)\left(u_{k t t}+\theta \hat{u}_{k t t}\right)-\nu^{\prime \prime \prime}(u) u_{t}^{2}-\nu^{\prime \prime}(u) u_{t t}\right] \hat{u}_{k} \\
& +2\left[\left(\nu^{\prime \prime}\left(u_{k}+\theta \hat{u}_{k}\right)\left(u_{k t}+\theta \hat{u}_{k t}\right)-\nu^{\prime \prime}(u) u_{t}\right] \hat{u}_{k t}\right. \\
& \left.\left.+\left[\nu^{\prime}\left(u_{k}+\theta \hat{u}_{k}\right)-\nu^{\prime}(u)\right] \hat{u}_{k t t}\right\} d \theta\right)^{2} d x d t
\end{aligned}
$$


We get

$$
\begin{aligned}
& \int_{Q_{T}}\left|\frac{\partial^{2}}{\partial t^{2}}\left[R\left(u_{k}, \hat{u}_{k}\right)\right](x, t)\right|^{2} d x d t \\
= & \int_{Q_{T}}\left(\int _ { 0 } ^ { 1 } \left\{\left[\nu^{\prime \prime \prime}\left(u_{k}+\theta \hat{u}_{k}\right)-\nu^{\prime \prime \prime}(u)\right]\left(u_{k t}+\theta \hat{u}_{k t}\right)^{2} \hat{u}_{k}\right.\right. \\
& +\nu^{\prime \prime \prime}(u)\left[\left(u_{k t}+\theta \hat{u}_{k t}\right)^{2}-u_{t}^{2}\right] \hat{u}_{k}+2\left[( \nu ^ { \prime \prime } ( u _ { k } + \theta \hat { u } _ { k } ) - \nu ^ { \prime \prime } ( u ) ] \left(u_{k t}\right.\right. \\
& \left.+\theta \hat{u}_{k t}\right) \hat{u}_{k t}+2 \nu^{\prime \prime}(u)\left(u_{k t}+\theta \hat{u}_{k t}-u_{t}\right) \hat{u}_{k t} \\
& +\left[\left(\nu^{\prime \prime}\left(u_{k}+\theta \hat{u}_{k}\right)-\nu^{\prime \prime}(u)\right]\left(u_{k t t}+\theta \hat{u}_{k t t}\right) \hat{u}_{k}\right. \\
& \left.\left.+\nu^{\prime \prime}(u)\left(u_{k t t}+\theta \hat{u}_{k t t}-u_{t t}\right) \hat{u}_{k}+\left[\nu^{\prime}\left(u_{k}+\theta \hat{u}_{k}\right)-\nu^{\prime}(u)\right] \hat{u}_{k t t}\right\} d \theta\right)^{2} d x d t \\
\leq & \int_{Q_{T}} \int_{0}^{1}\{\ldots\}^{2} d \theta d x d t=\int_{0}^{1}\{\ldots\}^{2} d x d t d \theta \\
\leq & 7\left[\int_{0}^{1} \int_{Q_{T}}\left|\nu^{\prime \prime \prime}\left(u_{k}+\theta \hat{u}_{k}\right)-\nu^{\prime \prime \prime}(u)\right|^{2}\left|\left(u_{k t}+\theta \hat{u}_{k t}\right)\right|^{2} \hat{u}_{k}^{2} d x d t d \theta\right. \\
& \left.+4 \int_{0}^{1} \int_{Q_{T}}\left|\nu^{\prime \prime}\left(u_{k}+\theta \hat{u}_{k}\right)-\nu^{\prime \prime}(u)\right|^{2} \mid u_{k t}+\theta \hat{u}_{k t}\right)\left.\right|^{2} \hat{u}_{k t}^{2} d x d t d \theta \\
& \left.+\int_{0}^{1} \int_{Q_{T}}\left|\nu^{\prime}\left(u_{k}+\theta \hat{u}_{k}\right)-\nu^{\prime}(u)\right|^{2} \hat{u}_{k t t}^{2} d x d t d \theta\right] \\
& +4 \int_{0}^{1} \int_{Q_{T}}\left|\nu^{\prime \prime}(u)\right|^{2}\left|u_{k t}+\theta \hat{u}_{k t}-u_{t}\right|^{2} \hat{u}_{k t}^{2} d x d t d \theta \\
& +\int_{0}^{1} \int_{Q_{T}}^{1}\left|\nu^{\prime \prime}\left(u_{k}+\theta \hat{u}_{k}\right)-\nu^{\prime \prime}(u)\right|^{2}\left|u_{k t t}+\theta \hat{u}_{k t t}\right|^{2} \hat{u}_{k}^{2} d x d t d \theta \\
& \\
& +\left.u\right|^{2}\left|u_{k t t}+\theta \hat{u}_{k t t}-u_{t t}\right|^{2} \hat{u}_{k}^{2} d x d t d \theta \\
& \\
& \\
& \\
&
\end{aligned}
$$


Thus we obtain

$$
\begin{aligned}
& \int_{Q_{T}}\left|\frac{\partial^{2}}{\partial t^{2}}\left[R\left(u_{k}, \hat{u}_{k}\right)\right](x, t)\right|^{2} d x d t \\
& \leq 7\left[\left\|\hat{u}_{k}\right\|_{C\left(Q_{T}\right)}^{2} \int_{0}^{1}\left(\int_{Q_{T}}\left|\nu^{\prime \prime \prime}\left(u_{k}+\theta \hat{u}_{k}\right)-\nu^{\prime \prime \prime}(u)\right|^{4} d x d t\right)^{\frac{1}{2}} \times\left(\int_{Q_{T}}\left|u_{k t}+\theta \hat{u}_{k t}\right|^{8} d x d t\right)^{\frac{1}{2}} d \theta\right. \\
& \left.+.\left\|\hat{u}_{k}\right\|_{C\left(Q_{T}\right)}^{2} \int_{0}^{1}\left(\int_{Q_{T}}\left|\nu^{\prime \prime \prime}(u)\right|^{4} d x d t\right)^{\frac{1}{2}} \times\left.\left(\int_{Q_{T}} \mid\left(u_{k t}+\theta \hat{u}_{k t}\right)^{2}-u_{t}^{2}\right)\right|^{4} d x d t\right)^{\frac{1}{2}} d \theta \\
& \left.+4\left\|\hat{u}_{k t}\right\|_{L_{4}\left(Q_{T}\right)}^{2} \sup _{(x, t) \in Q_{T}} \mid u_{k t}+\theta \hat{u}_{k t}\right)\left.\right|^{2} \times \int_{0}^{1}\left(\int_{Q_{T}}\left|\nu^{\prime \prime}\left(u_{k}+\theta \hat{u}_{k}\right)-\nu^{\prime \prime}(u)\right|^{4} d x d t\right)^{\frac{1}{2}} d \theta \\
& +4\left\|\hat{u}_{k t}\right\|_{L_{4}\left(Q_{T}\right)}^{2} \sup _{Q_{T}}\left|\nu^{\prime \prime}(u)\right|^{2} \int_{0}^{1}\left(\int_{Q_{T}}\left|u_{k t}+\theta \hat{u}_{k t}-u_{t}\right|^{4} d x d t\right)^{\frac{1}{2}} d \theta \\
& +\left\|\hat{u}_{k t}\right\|_{C\left(Q_{T}\right)}^{2} \int_{0}^{1}\left(\int_{Q_{T}}\left|\nu^{\prime \prime}\left(u_{k}+\theta \hat{u}_{k}\right)-\nu^{\prime \prime}(u)\right|^{4} d x d t\right)^{\frac{1}{2}} \cdot\left(\int_{Q_{T}}\left|u_{k t t}+\theta \hat{u}_{k t t}\right|^{4} d x d t\right)^{\frac{1}{2}} d \theta \\
& \left.+\left\|\hat{u}_{k}\right\|_{C\left(Q_{T}\right)}^{2} \int_{0}^{1}\left(\int_{Q_{T}}\left|\nu^{\prime \prime}(u)\right|^{4} d x d t\right)^{\frac{1}{2}}\left(\int_{Q_{T}}\left|u_{k t t}+\theta \hat{u}_{k t t}-u_{t t}\right|^{4} d x d t\right)^{\frac{1}{2}}\right) d \theta \\
& \left.+\int_{0}^{1}\left(\int_{Q_{T}}\left|\nu^{\prime}\left(u_{k}+\theta \hat{u}_{k}\right)-\nu^{\prime}(u)\right|^{4} d x d t\right)^{\frac{1}{2}}\left(\int_{Q_{T}}\left|\hat{u}_{k t t}\right|^{4} d x d t\right)^{\frac{1}{2}} d \theta\right] \\
& \leq c_{22}\left[\int_{0}^{1}\left(\int_{Q_{T}}\left|\nu^{\prime \prime \prime}\left(u_{k}+\theta \hat{u}_{k}\right)-\nu^{\prime \prime \prime}(u)\right|^{4} d x d t\right)^{\frac{1}{2}} \cdot\left\|\hat{u}_{k}\right\|_{C\left(Q_{T}\right)}^{2}\right. \\
& +\int_{0}^{1}\left(\int_{Q_{T}}\left|\left(u_{k t}+\theta \hat{u}_{k t}\right)^{2}-u_{t}^{2}\right|^{4} d x d t\right)^{\frac{1}{2}} d \theta \cdot\left\|\hat{u}_{k}\right\|_{C\left(Q_{T}\right)}^{2} \\
& +\int_{0}^{1}\left(\int_{Q_{T}}\left|\nu^{\prime \prime}\left(u_{k}+\theta \hat{u}_{k}\right)-\nu^{\prime \prime}(u)\right|^{4} d x d t\right)^{\frac{1}{2}} d \theta \cdot\left\|\hat{u}_{k}\right\|_{L_{4}\left(Q_{T}\right)}^{2} \\
& +\int_{0}^{1}\left(\int_{Q_{T}}\left|u_{k t}+\theta \hat{u}_{k t}-u_{t}\right|^{4} d x d t\right)^{\frac{1}{2}} d \theta \cdot\left\|\hat{u}_{k}\right\|_{C\left(Q_{T}\right)}^{2} \\
& +\int_{0}^{1}\left(\int_{Q_{T}}\left|\nu^{\prime \prime}\left(u_{k}+\theta \hat{u}_{k}\right)-\nu^{\prime \prime}(u)\right|^{4} d x d t\right)^{\frac{1}{2}} d \theta \cdot\left\|\hat{u}_{k}\right\|_{C\left(Q_{T}\right)}^{2} \\
& +\int_{0}^{1}\left(\int_{Q_{T}}\left|u_{k t t}+\theta \hat{u}_{k t t}-u_{t t}^{2}\right|^{4} d x d t\right)^{\frac{1}{2}} d \theta \cdot\left\|\hat{u}_{k}\right\|_{C\left(Q_{T}\right)}^{2} \\
& +\int_{0}^{1}\left(\int_{Q_{T}}\left|\nu^{\prime}\left(u_{k}+\theta \hat{u}_{k}\right)-\nu^{\prime}(u)\right|^{4} d x d t\right)^{\frac{1}{2}} d \theta \cdot\left\|\hat{u}_{k t t}\right\|_{L_{4}\left(Q_{T}\right)}^{2} .
\end{aligned}
$$


Let us note that, in view of (11) and (12), the last term can be estimated by $\left\|\hat{u}_{k}\right\|_{H^{4}\left(Q_{T}\right)}^{2}$. In view of Lebesgue theorem, the other terms are integrals converging to zero.

Indeed, $\left|\nu^{\prime \prime \prime}\left(u_{k}+\theta \hat{u}_{k}\right)-\nu^{\prime \prime \prime}(u)\right|^{4} \rightarrow 0$ almost everywhere with respect to $(x, t) \in$ $Q_{T}$ and $\left|\nu^{\prime \prime \prime}\left(u_{k}+\theta \hat{u}_{k}\right)-\nu^{\prime \prime \prime}(u)\right|^{4} \leq C_{23}$

for all $\theta \in[0,1]$. In view of (11) and (15), $\left|\left(u_{k t}+\theta \hat{u}_{k t}\right)^{2}-u_{t}^{2}\right|^{4} \rightarrow 0$ a.e. with respect to $(x, t) \in Q_{T}$ and $\left|\left(u_{k t}+\theta \hat{u}_{k t}\right)^{2}-u_{t}^{2}\right|^{4} \in L_{1}\left(Q_{T}\right)$ for all $\theta \in[0,1]$. In view of (11) and (15), $\left|u_{k t}+\theta \hat{u}_{k t}-u_{t}\right|^{4} \rightarrow 0$ a.e. with respect to $(x, t) \in Q_{T}$ and $\left|u_{k t}+\theta \hat{u}_{k t}-u_{t}\right|^{4} \in L_{1}\left(Q_{T}\right)$ for all $\theta \in[0,1]$. In view of (11) and (15), $\left|u_{k t t}+\theta \hat{u}_{k t t}-u_{t t}\right|^{4} \rightarrow$ 0 a.e. with respect to $(x, t) \in Q_{T}$ and, $\left|u_{k t t}+\theta \hat{u}_{k t t}-u_{t t}\right|^{4} \in L_{1}\left(Q_{T}\right)$ for all $\theta \in[0,1]$. In view of $(11)$ and $(15)\left|\nu^{\prime}\left(u_{k}+\theta \hat{u}_{k}\right)-\nu^{\prime}(u)\right|^{4} \rightarrow 0$ a.e. with respect to $(x, t) \in Q_{T}$

$$
\text { and }\left|\nu^{\prime}\left(u_{k}+\theta \hat{u}_{k}\right)-\nu^{\prime}(u)\right|^{4} \in L_{1}\left(Q_{T}\right) \text { for all } \theta \in[0,1] \text {. Similarly } \int_{Q_{T}}\left|\frac{\partial^{2}}{\partial x^{2}}\left[R\left(u_{k}, \hat{u}_{k}\right)\right](x, t)\right|^{2} d x d t
$$

can be estimated. In the same way, we are able to estimate the following terms:

$$
\int_{Q_{T}}\left|\frac{\partial^{2}}{\partial x \partial t}\left[R\left(u_{k}, \hat{u}_{k}\right)\right](x, t)\right|^{2} d x d t, \int_{Q_{T}}\left|\frac{\partial^{2}}{\partial t \partial x}\left[R\left(u_{k}, \hat{u}_{k}\right)\right](x, t)\right|^{2} d x d t, \int_{Q_{T}}\left|\frac{\partial^{3}}{\partial t^{3}}\left[R\left(u_{k}, \hat{u}_{k}\right)\right](x, t)\right|^{2} d x d t
$$

$\int_{Q_{T}}\left|\frac{\partial^{3}}{\partial x^{3}}\left[R\left(u_{k}, \hat{u}_{k}\right)\right](x, t)\right|^{2} d x d t, \int_{Q_{T}}\left|\frac{\partial^{3}}{\partial t^{2} \partial x}\left[R\left(u_{k}, \hat{u}_{k}\right)\right](x, t)\right|^{2} d x d t, \int_{Q_{T}}\left|\frac{\partial^{3}}{\partial t d x^{2}}\left[R\left(u_{k}, \hat{u}_{k}\right)\right](x, t)\right|^{2} d x d t$,

$\int_{Q_{T}}\left|\frac{\partial^{3}}{\partial t d x \partial t}\left[R\left(u_{k}, \hat{u}_{k}\right)\right](x, t)\right|^{2} d x d t, \int_{Q_{T}}\left|\frac{\partial^{3}}{\partial x d t \partial x}\left[R\left(u_{k}, \hat{u}_{k}\right)\right](x, t)\right|^{2} d x d t, \int_{Q_{T}}\left|\frac{\partial^{3}}{\partial x d t^{2}}\left[R\left(u_{k}, \hat{u}_{k}\right)\right](x, t)\right|^{2} d x d t$

and

$$
\int_{Q_{T}}\left|\frac{\partial^{3}}{\partial x^{2} d t}\left[R\left(u_{k}, \hat{u}_{k}\right)\right](x, t)\right|^{2} d x d t
$$

which all contain terms $\nabla^{\prime \prime \prime}(u)$ and $\nu^{(4)}(u)$. We then deduce (14). Hence, the operator $N$ is differentiable, and the proof of proposition is complete.

\section{Proof of the main result}

Consider the operator $\xi: H \longrightarrow H^{3}\left(Q_{T}\right)$ defined by $\xi(u)=L U+N U$. 
We know that $\xi$ is differentiable. Moreover, $\xi(0)=0$, and, since $\nu^{\prime}(0)=0, \xi^{\prime}(0)=$ $L \neq 0$.

Hence according to the theorem 1 and, by the inversion theorem, there exist open neighborhoods of zeros $U_{1} \subset H$ and $V \subset H^{3}\left(Q_{T}\right)$ such that the operator $\xi$ is $C^{1}$-diffeomorphism from $U_{1}$ to $V_{1}$; and the operaor $\eta=\xi^{-1}: V_{1} \longrightarrow U_{1}$ is also a $C^{1}$-diffeomorphism. The operater $P: H^{3}\left(Q_{T}\right) \times H^{3}\left(Q_{T}\right) \longrightarrow H$ defined by

$$
P[(f, g)](x, t)=\eta\left[\left(\frac{x}{l}-1\right) f(0)-\frac{x}{l} g(0)\right.
$$

is obviously differentiable on some neighborhood of zero in $H^{3}\left(Q_{T}\right) \times H^{3}\left(Q_{T}\right)$.Let $V_{2}$ be this neighlorhood $V_{2} \subset V_{1}$.

We wish to show the existence of $(f, g) \in V_{2}$ such that

$u(x, t)=\eta\left[\left(\frac{x}{l}-1\right) f(t)-\frac{x}{l} g(t)=P(f, g)(x, t)\right.$, which is a solution of $(6)-(8)$, also satisfies condition (9) of problem (6) - (9)

$$
u(x, T)=\chi_{1}(x) \Longrightarrow \frac{\partial u}{\partial t}(x, T)=\chi_{2}(T)
$$

and

$$
\frac{\partial u}{\partial t}(x, T)=\chi_{2}(T) \Longrightarrow \frac{\partial^{2} u}{\partial \chi^{2}}(x, T)=\frac{\partial^{2}}{\partial x^{2}} \chi_{2}(x)
$$

we have, using (2):

$$
F(x, T)-\frac{\partial^{2}}{\partial t^{2}}[P(f, g)](x, T)-N[P(f, g)](x, T)=-\frac{\partial^{2}}{\partial x^{2}} \chi_{1}(x)
$$

and

$$
\frac{\partial}{\partial t} F(x, T)-\frac{\partial^{3}}{\partial t^{3}}[P(f, g)](x, T)-\frac{\partial}{\partial t} N[P(f, \tilde{g})](x, T)=-\frac{\partial^{2}}{\partial x^{2}} \chi_{2}(x) .
$$

Consider the operators

$$
\begin{aligned}
& M_{1}: H^{3}(I) \times H^{3}(I) \longrightarrow H^{3}(\Gamma), \\
& M_{2}: H^{3}(I) \times H^{3}(I) \longrightarrow H^{3}(\Gamma) \\
& M_{1}(f, g)(x, T)=F(x, T)-\frac{\partial^{2}}{\partial t^{2}}[P(f, g)](x, T)-N[P(f, g)](x, T) \\
& M_{2}(f, g)(x, T)=\frac{\partial}{\partial t} F(x, T)-\frac{\partial^{3}}{\partial t^{3}}[P(f, g)](x, T)-\frac{\partial}{\partial t} N[P(f, g)](x, T) .
\end{aligned}
$$

It follows from foregoing results that $M_{1}$ and $M_{2}$ are differentiable; and straigthforevard calculation shows that

$M_{k}^{\prime}(0,0) \neq 0 \forall k$.

Hence, by the inversion theorem, open neighborhoods of zeros $U \subset U_{1} \subset H$ and $V \subset V_{2} \subset H^{3}(I) \times H^{3}(I)$ suth that the operator 
$M: H^{3}(I) \times H^{3}(I) \longrightarrow H^{3}(\Gamma) \times H^{3}(\Gamma)$ defined by

$M(f, g)=\left(M_{1}(f, g), M_{2}(f, g)\right)$ is also $C^{1}$-diffeomorphism from $V$ to $U$.

$\left(\chi_{1}, \chi_{2}\right) \in H^{5}(\Gamma) \times H^{4}(\Gamma) \Longrightarrow\left(\chi_{1}^{\prime \prime}, \chi_{2}^{\prime \prime}\right) \in H^{3}(\Gamma) \times H^{2}(\Gamma)$ And one can show quite straigthforwadly, by using Poicaré inequality, that, if $\left(\chi_{1}^{\prime \prime}, \chi_{2}^{\prime \prime}\right) \in H^{3}(\Gamma) \times H^{2}(\Gamma)$, then $\exists$ a constant $\gamma>0$ suth that

$$
\left\|\left(\chi_{1}^{\prime \prime}, \chi_{2}^{\prime \prime}\right)\right\|_{H^{3} \times H^{2}}<\gamma \Longrightarrow\left\{\begin{array}{l}
\left(\chi_{1}^{\prime \prime}, \chi_{2}^{\prime \prime}\right) \in V \subset V_{2} \\
\left(\chi_{1}, \chi_{2}\right) \in V \subset V_{2}
\end{array} .\right.
$$

Hence, $\forall\left(\chi_{1}, \chi_{2}\right) \in H^{5}(\Gamma) \cap H_{0}^{1}(\Gamma) \times H^{4}(\Gamma) \cap H_{0}^{1}(\Gamma)$, choose that constant $\gamma$ suth that $\left\|\left(\chi_{1}^{\prime \prime}, \chi_{2}^{\prime \prime}\right)\right\|_{H^{3} \times H^{2}}<\gamma$, so that $\exists$ a unique $(f, g) \in U \subset U_{1} \subset H$ suth that

$$
M(f, g)=\left(M_{1}(f, g), M_{2}(f, g)\right)=\left(-\frac{d^{2} \chi_{1}}{d x^{2}},-\frac{d^{2} \chi_{2}}{d x^{2}}\right) .
$$

Let

$$
u(x, t)=P(f, g)(x, t)=\eta\left[\left(\frac{x}{l}-1\right) f(t)-\frac{x}{l} g(t)\right]
$$

It's then enough to show that $u$ solves the problem $(6)-(9)$. This is quite straightforward, and the proof is complete.

It's obvious that $u$ satisfies (6) - (8), by definition of $\eta$. Let's show (9). We have: $M_{1}(f, g)(x, T)=-\frac{d^{2} \chi_{1}}{d x^{2}} \Longrightarrow$

$$
F(x, T)-\frac{\partial^{2}}{\partial t^{2}}[P(f, g)](x, T)+N[P(f, g)](x, T)=-\frac{d^{2} \chi_{1}}{d x^{2}}
$$

$P(f, g)$ is a solution $(6)-(8)$,we have

$$
\frac{\partial^{2}}{\partial t^{2}}[P(f, g)](x, t)-\frac{\partial^{2}}{\partial x^{2}}[P(f, g)](x, t)+N[P(f, g)](x, t)=F(x, t)
$$

and therefore, as $u(x, t)=P(f, g)(x, t)$,

$$
-\frac{\partial^{2} u}{\partial \chi^{2}}(x, T)=-\frac{d^{2} \chi_{1}}{d x^{2}}(x) \Longrightarrow u(x, T)=\chi_{1}(x)
$$

by (8) and since $\chi_{1} \in H_{0}^{1}(\Gamma)$. The other condition (9) is established in the same way. The proof is thus complete.

\section{References}

[1] V.A. Il'in V.V. Tikhomirov Volnovje ouravnjenje....."The wave equation with the Dirichlet boundary conditions"(Soviet Maths), Diffrerentialnie ouravnienie 35(5)(1999), 602-704 
[2] F. MOUKAMBA, D. AMPINI, N. YUMBA On local controlability of the quasilinear hyperbolic equation. Far East Journal of Mathemetical Sciences(FJMS).Volume 31,Issue 3,2008, p.393-407.

[3] MOUKAMBA F., AMPINI D. and YUMBA N. Local controlability of a nonlinear heat equation with integral constraint Int. Journal of Math. Analysis, Vol 6, 2012, No 59, 2927-2942. (To be appear)

[4] Mikaïlov V.P. P.D.E. MOSCOU 1976.

Received: October, 2012 